\title{
A Retrospective Study Investigating Risks of Acute Respiratory Distress Syndrome and Mortality Following Human Metapneumovirus Infection in Hospitalized Adults
}

\author{
Hyunjung Hwang, Yujin Kim, Jeong-Woong Park, Sung Hwan Jeong, and Sun Young Kyung \\ Division of Pulmonology, Allergy and Critical Care, Department of Internal Medicine, Gachon University Gill Medical Center, Incheon, Korea
}

Background: Human metapneumovirus (hMPV) is a relatively recently identified respiratory virus that induces respiratory symptoms similar to those of respiratory syncytial virus infection in children. The characteristics of hMPV-infected adults are unclear because few cases have been reported.

Methods: We conducted a retrospective review of hospitalized adult patients with a positive multiplex real-time polymerase chain reaction assay result from 2012 to 2016 at a single tertiary referral hospital in South Korea. We analyzed clinical characteristics of the enrolled patients and divided patients into an acute respiratory distress syndrome (ARDS) group and a non-ARDS group.

Results: In total, 110 adults were reviewed in this study. Their mean age was 61.4 years, and the majority $(n=105,95.5 \%)$ had comorbidities or were immunocompromised. Most of the patients had pneumonia on chest X-ray $(n=88,93.6 \%), 22(20.0 \%)$ had ARDS, and $12(10.9 \%)$ expired during hospitalization. The mortality rate for patients with ARDS was higher than that of the other patients (36.4\% vs. $5.7 \%, P=0.001$ ). The risk factor for hMPV-associated ARDS was heart failure (odds ratio, $5.24 ; P=0.044$ ) and laboratory values were increased blood urea nitrogen and increased C-reactive protein. The acquisition site of infection was divided into community vs. nosocomial; 43 patients (39.1\%) had a nosocomial infection. The risk factors for nosocomial infection were an immunocompromised state, malignancy and immunosuppressive treatment.

Conclusions: These data suggest that hMPV is one of the important respiratory pathogens important respiratory pathogen that causes pneumonia/ARDS in elderly, immunocompromised individuals and that it may be transmitted via the nosocomial route.

Key Words: metapneumovirus; mortality; nosocomial infection; respiratory distress syndrome, adult.

\section{Introduction}

Human metapneumovirus (hMPV) was first identified in 2001, the Netherlands from a pediatric patient who had symptoms similar to those of respiratory syncytial virus (RSV) infection. It is a member of the paramyxovirus family and is genetically similar to RSV [1]. Typically hMPV infections occur between March and April, and account for 7\% of respiratory tract infections [2].

A hMPV infection commonly occurs in children less than 2 years old and manifests as mild flu-like symptoms, similar

Received on August 17, 2016 Revised on March 1, 2017 Accepted on March 16, 2017

Correspondence to: Sun Young Kyung, Division of Pulmonology, Allergy and Critical Care, Department of Internal Medicine, Gachon University Gil Medical Center, 21 Namdong-daero 774beon-gil, Namdong-gu, Incheon 21565, Korea

Tel: +82-32-460-2634, Fax: +82-32-469-4320, E-mail: light@gilhospital.com

*No potential conflict of interest relevant to this article was reported.

(cc) This is an Open Access article distributed under the terms of the Creative Commons Attribution Non-Commercial License (http://creativecommons.org/ licenses/by-nc/4.0/) which permits unrestricted non-commercial use, distribution, and reproduction in any medium, provided the original work is properly cited. Copyright (c) 2017 The Korean Society of Critical Care Medicine 
to RSV [3]. Furthermore, hMPV is a major contributor to the burden of wintertime respiratory illness in older adults that is peak incidence at 65 years of age and immunocompromised individuals $[2,4,5]$. hMPV infections in children are usually mild and self-limiting, but in elderly and immunocompromised patients, the clinical course can progress to acute respiratory distress syndrome (ARDS) [6]. Studies of patients with hMPV who develop severe illness have focused on children; few have involved adults $[7,8]$. Nosocomial infection has been reported in several studies as a mode of transmission [5,9-11]. Nosocomial hMPV infection of adults occurs predominantly in human immunodeficiency virus-infected persons [12].

There are few studies on hMPV infection of adults in Korea. And the number of immunocompromised patients is increasing in hospitals. These patients are also vulnerable to previously neglected pathogens. Therefore, we designed a retrospective review of hMPV-infected adults. The clinical characteristics of the patients-including demographic data, comorbidities, presence of pneumonia or ARDS, acquisition site (community-acquired or nosocomial), and risk factors for ARDS — were reviewed.

\section{Materials and Methods}

This study was a retrospective review of medical records; institutional review board approval was obtained (No. GAIRB2016-179). We identified all patients at Gachon University Gil Medical Center, a tertiary referral hospital, with a laboratory-confirmed hMPV infection as diagnosed by positive multiplex real time-polymerase chain reaction (mRT-PCR) between January 2012 and April 2016. Nasopharyngeal or oropharyngeal samples were obtained in the emergency department, in an inpatient setting, or in an outpatient setting. All diagnostic testing was performed at the Gil Medical Center Laboratory Department. Multiplex real time-PCR was performed using Anyplex II RV16 Detection kit (Seegene, Seoul, Korea). This kit detected in parallel for the following 16 respiratory viruses: human bocavirus, human enterovirus, influenza virus A and B, parainfluenza virus 1, 2, 3, and 4, RSV A and B, coronavirus OC43, 229E, NL63, human rhinovirus $\mathrm{A} / \mathrm{B} / \mathrm{C}$, and metapneumovirus. A total of 652 cases of hMPV infection were identified by mRT-PCR from nasopharyngeal or oropharyngeal swabs among patients. We collected hospitalized adult patients over 18 years with positive hMPV mRT-PCR assay result among 652 patients. The 110 of hospitalized adult patients with hMPV infection were included in the primary analysis.

Demographic, comorbidity, and hospitalization data of 110 adult patients were collected. The data included chronic respiratory disease, cardiac disease, immunocompromised state, end-stage renal disease (ESRD) requiring dialysis, cirrhosis, corticosteroid use, and immunosuppressive therapy. Viral co-infection was defined as identification by mRT-PCR from a nasopharyngeal or oropharyngeal swab. Patients who had either a positive blood culture or a positive sputum culture from a satisfactory specimen were identified as having bacterial co-infection. We reviewed the medical records of the patients to identify those admitted to the intensive care unit (ICU) with a nosocomial infection and who met the criteria for ARDS using the Berlin definition [13]. The incubation period is estimated to be 4 to 6 days [14]. Nosocomial infection was defined as an infection that occurred up to 5 days after discharge or more than 5 days in admission. Death information of patients was confirmed using hospital medical record and obliterated information of national health insurance service which means death.

Clinically relevant parameters were identified on the day of execution of mRT-PCR and admission to the ICU or ward; these included temperature, respiratory rate, applied fraction of inspired oxygen, partial pressure of arterial oxygen, white blood cell count, blood urea nitrogen $(\mathrm{BUN}) /$ creatinine, total bilirubin, $\mathrm{C}$-reactive protein (CRP) level, and procalcitonin level. The patients were divided into non-ARDS and ARDS groups, and the risk factors and laboratory values for ARDS were assessed.

\section{1) Statistical analysis}

All data analyses were performed using SPSS version 
20.0 (IBM Corp., Armonk, NY, USA). Categorical variables were compared by Fisher exact test, and continuous variables by Wilcoxon's rank-sum test. The impact of potential risk factors on the development of ARDS was analyzed with univariate logistic regression analysis. Statistically significant variables at univariate analysis were included into a multivariate logistic regression analysis with backward elimination to identify independent risk factors of ARDS. The independent influences of risk factors for ARDS were expressed as the odds ratio (OR) with their $95 \%$ confidence intervals. Significance was taken as $\mathrm{P}<0.05$.

\section{Results}

\section{1) Characteristics of adults with a hMPV infection}

The mean age of the adult patients was $61.4 \pm 16.6$ years, and there was no difference according to sex (Table 1). Overall, 19 patients $(17.3 \%)$ had viral co-infections, and $22(20.0 \%)$ had bacterial co-infections. Co-infection did not affect event of ARDS, nosocomial infection and mortality. Most patients $(\mathrm{n}=105,95.5 \%)$ had comorbidities; these included diabetes, malignancy, pulmonary disease, cardiac disease, ESRD, and liver cirrhosis and they were being treated with corticosteroid or immuno-

Table 1. Characteristics of hMPV-infected patients according to ARDS or non-ARDS group

\begin{tabular}{|c|c|c|c|c|}
\hline & All & Non-ARDS & ARDS & $\mathrm{P}$-value \\
\hline No. of patients & 110 & 88 & 22 & \\
\hline Age (yr) & $61.4 \pm 16.6$ & $59.8 \pm 16.6$ & $68.0 \pm 15.2$ & 0.036 \\
\hline Male sex & $46(41.8)$ & $36(40.9)$ & $10(45.5)$ & 0.439 \\
\hline \multicolumn{5}{|l|}{ Co-infection } \\
\hline Virus & $19(17.3)$ & $17(19.3)$ & $2(9.1)$ & 0.211 \\
\hline Bacteria & $22(20.0)$ & $15(17.0)$ & $7(31.8)$ & 0.108 \\
\hline \multicolumn{5}{|l|}{ Comorbidity } \\
\hline Solid tumor & $19(17.3)$ & $16(18.2)$ & 3 (13.6) & 0.681 \\
\hline Hematologic disorder & $10(9.1)$ & $8(9.1)$ & $2(9.1)$ & 0.442 \\
\hline $\mathrm{DM}$ & $29(26.4)$ & $21(23.9)$ & $8(36.4)$ & 0.178 \\
\hline Asthma & $12(10.9)$ & $10(11.4)$ & $2(9.1)$ & 0.555 \\
\hline COPD & $12(10.9)$ & $9(10.2)$ & $3(13.6)$ & 0.445 \\
\hline$\| \mathrm{LD}$ & $2(1.8)$ & $1(1.1)$ & $1(4.5)$ & 0.361 \\
\hline ESRD & $7(6.4)$ & $4(4.5)$ & $3(13.6)$ & 0.141 \\
\hline LC & $5(4.5)$ & $5(5.7)$ & 0 & 0.20 \\
\hline KT & $3(2.7)$ & $3(3.4)$ & 0 & 0.508 \\
\hline $\mathrm{CHF}$ & $7(6.3)$ & $3(3.4)$ & $4(18.2)$ & 0.029 \\
\hline $\mathbb{H D}$ & $4(3.6)$ & $2(2.3)$ & $2(9.1)$ & 0.178 \\
\hline Steroid used ${ }^{\mathrm{a}}$ & $15(13.6)$ & $13(14.8)$ & $2(9.1)$ & 0.383 \\
\hline Immunotherapy ${ }^{b}$ & $17(15.5)$ & $14(15.9)$ & 3 (13.6) & 0.545 \\
\hline Nosocomial infection & $43(39.1)$ & $36(40.9)$ & $7(31.8)$ & 0.299 \\
\hline Pneumonia & 103 (93.6) & $83(94.3)$ & $20(90.9)$ & 0.560 \\
\hline In-hospital mortality & $12(10.9)$ & $5(5.7)$ & $8(36.4)$ & 0.001 \\
\hline 1-Year all-cause mortality & $17(15.5)$ & $9(10.2)$ & $9(40.9)$ & 0.002 \\
\hline
\end{tabular}

Values are presented as mean \pm standard deviation or number (\%).

hMPV: human metapneumovirus; ARDS: acute respiratory distress syndrome; DM: diabetes mellitus; COPD: chronic obstructive pulmonary disease; ILD: interstitial lung disease; ESRD: end-stage renal disease; LC: liver cirrhosis; KT: kidney transplantation; CHF: congestive heart failure; IHD: ischemic heart disease.

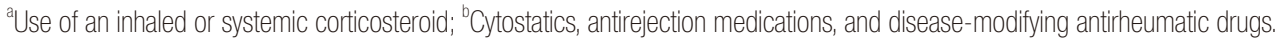


Table 2. Laboratory parameters of hMPV-infected patients according to non-ARDS and ARDS group

\begin{tabular}{|c|c|c|c|c|}
\hline & All & Non-ARDS & ARDS & P-value \\
\hline No. of patients & 110 & 88 & 22 & \\
\hline Hemoglobin (g/dl) & $11.4 \pm 2.2$ & $11.6 \pm 2.1$ & $10.6 \pm 2.5$ & 0.053 \\
\hline Platelet $\left(\times 10^{3} / \mathrm{mm}^{3}\right)$ & $195.7 \pm 92.9$ & $195.5 \pm 96.2$ & $196.5 \pm 80.5$ & 0.967 \\
\hline WBC count $\left(\times 10^{3} / \mathrm{mm}^{3}\right)$ & $8.1(9.6)$ & $8.3(10.2)$ & $6.3(9.2)$ & 0.791 \\
\hline Lymphocytes (/mm³) & $913.8(961.1)$ & $983.2(851.2)$ & $638.8(1,971.6)$ & 0.019 \\
\hline BUN (mg/dl) & $16.4(21.8)$ & $14.6(15.7)$ & $35.5(35.9)$ & 0.002 \\
\hline $\mathrm{Cr}(\mathrm{mg} / \mathrm{dl})$ & $0.85(0.7)$ & $0.8(0.6)$ & $1.0(2.4)$ & 0.023 \\
\hline Total bilirubin (mg/dl) & $0.65(0.6)$ & $0.7(0.6)$ & $0.5(0.4)$ & 0.247 \\
\hline Albumin (g/dl) & $3.5 \pm 0.6$ & $3.6 \pm 0.6$ & $3.1 \pm 0.6)$ & 0.002 \\
\hline $\mathrm{CRP}$ (mg/dl) & $9.48 \pm 8.5$ & $7.8 \pm 7.3$ & $16.1 \pm 9.6$ & 0.001 \\
\hline Procalcitonin (ng/ml) & $0.83(2.31)$ & $0.57(1.15)$ & $2.35(6.57)$ & 0.165 \\
\hline
\end{tabular}

Values are presented as mean \pm standard deviation or median (interquartile range).

hMPV: human metapneumovirus; ARDS: acute respiratory distress syndrome; WBC: white blood cell; BUN: blood urea nitrogen; Cr: serum creatinine; CRP: C-reactive protein.

Table 3. Risk factors for ARDS among patients with hMPV infection

\begin{tabular}{|c|c|c|c|c|c|c|}
\hline \multirow{2}{*}{ Variable } & \multicolumn{3}{|c|}{ Univariate analysis } & \multicolumn{3}{|c|}{ Multivariate analysis } \\
\hline & $\mathrm{OR}$ & $95 \% \mathrm{Cl}$ & P-value & $\mathrm{OR}$ & $95 \% \mathrm{Cl}$ & P-value \\
\hline Age & 1.034 & $1.00-1.07$ & 0.040 & 1.031 & $0.99-1.07$ & 0.070 \\
\hline CHF & 6.296 & $1.30-30.60$ & 0.023 & 5.249 & $1.04-26.39$ & 0.044 \\
\hline
\end{tabular}

Risk factor analysis (Nagelkerke $R^{2}$ value $=0.120$, Hosmer and Lemeshow goodness of fit with a $\chi^{2}$ value $=0.762$ ).

ARDS: acute respiratory distress syndrome; hMPV: human metapneumovirus; OR: odds ratio; Cl: confidence interval; CHF: congestive heart failure.

suppressive medication. Almost patients had pneumonia on chest X-ray (93.6\%), and 22 patients (20\%) had ARDS (Table 1). Half of the patients with hMPV-associated ARDS had severe disease according to the Berlin definition. The in-hospital mortality rate was $10.9 \%$, and the 1-year allcause mortality rate was $15.5 \%$. The patients with ARDS showed higher in-hospital (36.4\%) and 1-year all-cause (40.9\%) mortality rates than those without ARDS (Table 1). Forty-three patients were found to have nosocomial infections (39\%) (Table 1). Regarding laboratory findings, lymphocytopenia $\left(<1,500 / \mathrm{mm}^{3}\right)$, high levels of CRP, BUN and creatinine, and low levels of albumin were observed (Table 2).

\section{2) Risk factors for hMPV-associated ARDS}

The non-ARDS group included 88 patients while the ARDS group included 22 patients. The mean age of the non-ARDS group was 59.8 years, while that of the ARDS group was 68.0 years $(\mathrm{OR}, 1.034 ; \mathrm{P}=0.040)$. However, multivariate logistic regression showed age was not significant (Table 3). The pattern of viral and bacterial co-infection did not differ between the ARDS and nonARDS groups (Table 1). The rates of comorbidities in the hMPV-associated ARDS patients were similar in hMPVassociated ARDS patients, with the exception of congestive heart failure (OR, 5.249; $\mathrm{P}=0.044)$ (Table 3$)$. The inhospital and 1-year all-cause mortality rates of the ARDS patients were $36.4 \%$ and $40.9 \%$, respectively (Table 1). Additional analysis showed Kendal rank correalation coefficient was $0.752(\mathrm{P}=0.001)$ between in hospital mortality and 1-year mortality. Therefore 1-year all-cause mortality was not independent variable.

\section{3) Characteristics of nosocomial hMPV infection}

Overall, 67 patients had community-acquired hMPV infections and 43 had nosocomial infections. The patients 
with nosocomial infection showed the presence of comorbidities (e.g., solid tumor, hematologic disorder, liver cirrhosis, and immunosuppressive therapy; $\mathrm{P}<0.05$ ). However, the rates of mortality and hMPV-associated ARDS were not different between the community-acquired and nosocomial infection groups (Table 4).

Table 4. Characteristics of hMPV-infected patients according to acquisition site: community-acquired or nosocomial infection

\begin{tabular}{|c|c|c|c|}
\hline & $\begin{array}{l}\text { Community- } \\
\text { acquired }\end{array}$ & Nosocomial & P-value \\
\hline No. of patients & 67 & 43 & \\
\hline Age (yr) & $63.5 \pm 17.1$ & $58.3 \pm 15.5$ & 0.113 \\
\hline Male sex & $28(41.8)$ & $18(41.9)$ & 0.575 \\
\hline \multicolumn{4}{|l|}{ Co-infection } \\
\hline Virus & $9(13.4)$ & $10(23.3)$ & 0.142 \\
\hline Bacteria & $13(19.4)$ & $9(20.9)$ & 0.515 \\
\hline \multicolumn{4}{|l|}{ Comorbidity } \\
\hline Solid tumor & $6(9.0)$ & $13(30.2)$ & 0.005 \\
\hline Hematologic disorder & $1(1.5)$ & $9(20.9)$ & 0.001 \\
\hline $\mathrm{DM}$ & 18 (26.9) & $11(25.6)$ & 0.532 \\
\hline Asthma & $10(14.9)$ & $2(4.7)$ & 0.081 \\
\hline COPD & $9(13.4)$ & $3(7.0)$ & 0.231 \\
\hline$\| \mathrm{LD}$ & $2(3.0)$ & 0 & 0.369 \\
\hline ESRD & $4(6.0)$ & $3(7.0)$ & 0.564 \\
\hline LC & 0 & $5(11.6)$ & 0.008 \\
\hline $\mathrm{KT}$ & $1(1.5)$ & $2(4.7)$ & 0.338 \\
\hline CHF & $6(9.0)$ & $1(2.3)$ & 0.162 \\
\hline $\mathbb{H D}$ & $2(3.0)$ & $2(4.7)$ & 0.510 \\
\hline Steroid used ${ }^{\mathrm{a}}$ & $11(16.4)$ & $2(9.3)$ & 0.221 \\
\hline $\begin{array}{l}\text { Immunosuppressive } \\
\text { therapy }^{b}\end{array}$ & $4(6.0)$ & $13(30.2)$ & 0.001 \\
\hline Initial pneumonia & $61(91.1)$ & $42(97.7)$ & 0.166 \\
\hline ARDS & $15(22.4)$ & $7(16.3)$ & 0.299 \\
\hline In-hospital mortality & $8(11.9)$ & $5(11.6)$ & 0.606 \\
\hline $\begin{array}{l}\text { 1-Year all-cause } \\
\text { mortality }\end{array}$ & $8(11.9)$ & $10(23.3)$ & 0.098 \\
\hline
\end{tabular}

Values are presented as mean \pm standard deviation or number (\%). hMPV: human metapneumovirus; DM: diabetes mellitus; COPD: chronic obstructive pulmonary disease; ILD: interstitial lung disease; ESRD: end-stage renal disease; LC: liver cirrhosis; KT: kidney transplantation; CHF: congestive heart failure; IHD: ischemic heart disease.

a Use of an inhaled or systemic corticosteroid; 'Cytostatics, antirejection medications, and disease-modifying antirheumatic drugs.

\section{Discussion}

Our study is the first to characterize ARDS and mortality in the adult patients with hMPV infections in Korea. This study evaluated the clinical features of 110 hospitalized adults with a positive hMPV mRT-PCR assay result at a tertiary referral hospital in South Korea from 2012 to 2016. This was a retrospective study of hMPV-infected adults and provided detailed clinical data, including comorbidities, radiologic findings, and laboratory findings, as well as the demographic data of the subjects. Most of the adults with hMPV infection had pneumonia on chest $\mathrm{X}$-ray, and $20 \%$ had ARDS. The in-hospital mortality rate of ARDS patients was $36.4 \%$, and $10.9 \%$ of all patients died during hospitalization. Nosocomial infection occurred in $39.1 \%$ of the subjects. Other study shows fortyeight percent of ICU patient is ARDS and mortality is $18 \%$ [8]. Although the scope of the studies, it is necessary to focus on high ARDS incidence and high mortality rates.

The increasing availability and broadened scope of viral respiratory polymerase chain reaction panels helps our understanding of viral pathogens. The mRT-PCR assay had a sensitivity and specificity higher than $88 \%$ and $98.6 \%$. And the mRT-PCR could detect co-infecting respiratory viruses, even at low viral loads that cannot be detected using culture techniques [15].

During the study period, the peak incidence of hMPV infection was in April, after that of influenza which had peak in February. A study in a similar area conducted from 2000 to 2005 reported a peak incidence of hMPV infection in January to March [16], and another in March to April [17]. In this study, $17 \%$ of the subjects had viral co-infections, most frequently with influenza virus (Table 4). This is in agreement with a previous report [4]. These co-infections may be due to the overlap of the periods of peak incidence of these viruses. hMPV infections occur between March and April. And RSV infection is in December and Influenza is in January and February (Supplementary Figure 1). A high percentage (90\% to $100 \%$ ) of children in 5-10 years old have been infected. $78 \%$, un- 
der 5 years of age in this study (Supplementary Figure 2).

The mean age of the adult patients was 61.4 years, and all but five had comorbidities such as diabetes, malignancy, airway obstructive diseases, ESRD, and heart failure. Such comorbidities and old age can result in an immunocompromised state, possibly leading to the development of severe viral infections such as pneumonitis or ARDS. hMPV infections in healthy adults usually show mild clinical features from asymptomatic to upper respiratory symptoms, similar to RSV infection [18]. However, frail elderly patients with an hMPV infection could progress to pneumonitis, ARDS, or death $[8,12,18]$. In this study, severe hMPV infection was associated with a risk of pneumonia, ARDS, and mortality in immunocompromised elderly patients. The findings of this study suggest that $20 \%$ of hospitalized adults with an hMPV infection will develop ARDS. The risk factor for hMPV-associated ARDS was congestive heart failure. It is known that viral infection is one of the risk factors for aggravation of congestive heart failure. When patients with congestive heart failure are infected by hMPV, they have risks both aggravation of congestive heart failure and ARDS [8]. The patients with ARDS and congestive heart failure may also have had pulmonary edema; however, this could not be determined because of the limitations inherent to a retrospective study. The patients with ARDS among hMPVinfected adults showed lymphocytopenia, higher levels of BUN, creatinine, and CRP and lower levels of albumin compared to non-ARDS patients. In-hospital mortality of severe ARDS (OR, 9.48) was higher than ARDS (OR, 5.24). The in-hospital mortality rate of the patients with hMPV infection was $10.9 \%$. The in-hospital mortality rate of the ARDS group (36.4\%) was significantly higher than that of the non-ARDS group (5.7\%). The risk factors for hMPV-associated ARDS should be considered as congestive heart failure. These results suggest that hMPV is an important and previously underappreciated cause of ARDS in adult patients. Severe clinical manifestations such as ARDS should be considered when hMPV infection is diagnosed in adult patients with congestive heart failure.
The hMPV infection has been reported in immunocompromised patients, including lung transplant and hematopoietic stem cell transplant recipients [3,19]. Moreover, outbreaks in healthcare facilities have been reported $[2,9]$. When considering the implications of these findings, it should be noted that the risk of nosocomial hMPV infection is on the rise among patients with blood cancer or patients with a solid tumor [20] who were recently admitted for chemotherapy. The additional analysis showed that immunosuppressive therapy is interaction variable with cancer. A patient with liver cirrhosis was not explained as risk factor of nosocomial infection because small cases and a further research is needed. Such patients typically reside in shared rooms containing four to six patients. Therefore, the possibility of hMPV infection should be considered in long-term immunocompromised patients residing in shared rooms. Droplet respiratory precautions are appropriate for preventing the transmission of hMPV, particularly in immunocompromised patients.

Table 5. Etiologies of co-infection in patients with hMPV infection

\begin{tabular}{lll}
\hline & No. & \multicolumn{1}{c}{ Confirmed species (no.) } \\
\hline Bacteria & & \\
Pneumonia & 5 & S.pneumoniae (2) \\
VAP & 2 & K.pneumoniae (1) \\
Sepsis & 3 & E.coli (1) / Chlamydiae (1) \\
CRBSI & 2 & MRSA (3) \\
Colits & 2 & C. difficile (2) \\
UTI & 2 & \\
Liver abscess & 1 & K. pneumoniae (1) \\
Wound infection & 4 & \\
Virus & & \\
Coronavirus & 5 & \\
Influenza A & 5 & \\
Influenza B & 1 & \\
Adenovirus & 2 & \\
Rhinovirus & 2 & \\
Cytomegalovirus & 2 & \\
RSV & 1 & \\
Bocavirus & 1 & \\
\hline
\end{tabular}

hMPV: human metapneumovirus; VAP: ventilator associated pneumonia; CRBSI: catheter related bloodstream infection; MRSA: methicillin resistant StaphylococcuS aureus; UTI: urinary tract infection; RSV: respiratory syncytial virus. 
This study has multiple limitations. Whether hMPV was the pathogen in all patients enrolled in this study was unclear. However, the mRT-PCR assay used has high sensitivity and specificity. Also, the rate of co-infection with other pathogens was not high (Table 5). Future studies should assess the clinical features of hMPV infection in adults, determine the clinical potential of the virus as a respiratory pathogen to induce severe ARDS, and develop novel antiviral agents or vaccines.

In conclusion, this study suggests that hMPV is an important respiratory pathogen that causes pneumonia/ ARDS in elderly, immunocompromised individuals and that it is transmitted via the nosocomial route. Clinicians need to consider mMPV virus and sufficient attention is required.

\section{ORCID}

Hyunjung Hwang http://orcid.org/0000-0003-3688-0962

\section{Supplementary Materials}

The online-only Supplement data are available with this article online: https://doi.org/10.4266/kjccm.2017.00038.

\section{References}

1. van den Hoogen BG, de Jong JC, Groen J, Kuiken T, de Groot R, Fouchier RA, et al. A newly discovered human pneumovirus isolated from young children with respiratory tract disease. Nat Med 2001;7:719-24.

2. Panda S, Mohakud NK, Pena L, Kumar S. Human metapneumovirus: review of an important respiratory pathogen. Int J Infect Dis 2014;25:45-52.

3. Williams JV, Harris PA, Tollefson SJ, Halburnt-Rush LL, Pingsterhaus JM, Edwards KM, et al. Human metapneumovirus and lower respiratory tract disease in otherwise healthy infants and children. N Engl J
Med 2004;350:443-50.

4. Walsh EE, Peterson DR, Falsey AR. Human metapneumovirus infections in adults: another piece of the puzzle. Arch Intern Med 2008;168:2489-96.

5. Seo S, Gooley TA, Kuypers JM, Stednick Z, Jerome KR, Englund JA, et al. Human metapneumovirus infections following hematopoietic cell transplantation: factors associated with disease progression. Clin Infect Dis 2016;63:178-85.

6. Contentin L, Guillon A, Garot D, Gaudy-Graffin C, Perrotin D. Acute respiratory distress syndrome secondary to human metapneumovirus infection in a young healthy adult. Intensive Care Med 2013;39:533-4.

7. Choi SH, Hong SB, Ko GB, Lee Y, Park HJ, Park SY, et al. Viral infection in patients with severe pneumonia requiring intensive care unit admission. Am J Respir Crit Care Med 2012;186:325-32.

8. Hasvold J, Sjoding M, Pohl K, Cooke C, Hyzy RC. The role of human metapneumovirus in the critically ill adult patient. J Crit Care 2016;31:233-7.

9. Osbourn M, McPhie KA, Ratnamohan VM, Dwyer DE, Durrheim DN. Outbreak of human metapneumovirus infection in a residential aged care facility. Commun Dis Intell Q Rep 2009;33:38-40.

10. Boivin G, De Serres G, Hamelin ME, Côté S, Argouin M, Tremblay G, et al. An outbreak of severe respiratory tract infection due to human metapneumovirus in a long-term care facility. Clin Infect Dis 2007;44:1152-8.

11. Liao RS, Appelgate DM, Pelz RK. An outbreak of severe respiratory tract infection due to human metapneumovirus in a long-term care facility for the elderly in Oregon. J Clin Virol 2012;53:171-3.

12. Groome MJ, Moyes J, Cohen C, Walaza S, Tempia $\mathrm{S}$, Pretorius M, et al. Human metapneumovirus-associated severe acute respiratory illness hospitalisation in HIV-infected and HIV-uninfected South African children and adults. J Clin Virol 2015;69:125-32.

13. ARDS Definition Task Force, Ranieri VM, Rubenfeld GD, Thompson BT, Ferguson ND, Caldwell E, et al. Acute respiratory distress syndrome: the Berlin 
Definition. JAMA 2012;307:2526-33.

14. Peiris JS, Tang WH, Chan KH, Khong PL, Guan Y, Lau YL, et al. Children with respiratory disease associated with metapneumovirus in Hong Kong. Emerg Infect Dis 2003;9:628-33.

15. Kim HK, Oh SH, Yun KA, Sung H, Kim MN. Comparison of Anyplex II RV16 with the xTAG respiratory viral panel and Seeplex RV15 for detection of respiratory viruses. J Clin Microbiol 2013;51:1137-41.

16. Choi EH, Lee HJ, Kim SJ, Eun BW, Kim NH, Lee $\mathrm{JA}$, et al. The association of newly identified respiratory viruses with lower respiratory tract infections in Korean children, 2000-2005. Clin Infect Dis 2006; 43:585-92.

17. Mizuta K, Abiko C, Aoki Y, Ikeda T, Matsuzaki Y, Itagaki $\mathrm{T}$, et al. Seasonal patterns of respiratory syn- cytial virus, influenza A virus, human metapneumovirus, and parainfluenza virus type 3 infections on the basis of virus isolation data between 2004 and 2011 in Yamagata, Japan. Jpn J Infect Dis 2013;66:140-5.

18. Haas LE, Thijsen SF, van Elden L, Heemstra KA. Human metapneumovirus in adults. Viruses 2013;5: 87-110.

19. Dokos C, Masjosthusmann K, Rellensmann G, Werner C, Schuler-Lüttmann S, Müller KM, et al. Fatal human metapneumovirus infection following allogeneic hematopoietic stem cell transplantation. Transpl Infect Dis 2013;15:E97-101.

20. Kamboj M, Gerbin M, Huang CK, Brennan C, Stiles $\mathrm{J}$, Balashov S, et al. Clinical characterization of human metapneumovirus infection among patients with cancer. J Infect 2008;57:464-71. 